are made up of normal movements, carefully thought out to avoid muscular over-development and strain and to develop control, strength, and grace. Repose, relaxation, and rhythm are considered to be of great importance, all of which are necessary to counteract the effects of modern living. This form of exercise should be of benefit to any type of person, especially to neurotic subjects, as it possesses so much rhythm and teaches the expression of individuality. The classes are graded to suit both the mental and physical ability of pupils, and all the teachers are people of education and culture with a knowledge of anatomy.

\section{Medical Work in Newfoundland}

The great diversity of the medical and other philanthropic work carried on by the Grenfell Association of Great Britain and Ireland is well illustrated by its annual report for the year ending September 30, 1937. The St. Anthony Hospital, which began to function in 1902, dealt with 830 patients in the year under review, the majority of these requiring general surgical treatment and medical care for conditions other than tuberculosis. Cases of pulmonary tuberculosis numbered 49 , of malnutrition 45 , and of surgical tuberculosis 20 ; while there were 75 obstetrical cases, including 36 normal and 21 operative deliveries. Beriberi, of which there was a large number of cases for many years, is rapidly decreasing, in spite of the fact that the economic situation of the population of Labrador and Northern Newfoundland is probably worse than it has been for a long time. Many more families have gardens now, and educational work regarding diet has been continuing steadily. The Government has also insisted that all persons receiving relief shall use a more nutritive flour instead of white flour. The increase in tuberculous cases is partly accounted for by earlier diagnosis. The most serious problem is whether the hospital should assume the treatment of the more hopeless chronic cases, each of which costs the institution several thousand dollars a year. The St. Anthony Hospital provides treatment for the population of an immense area extending from Cape St. John on the east coast of Newfoundland to Port Saunders on the west coast and along the Strait of Belle Isle. There are two Government nurses in White Bay, one Grenfell Mission nurse at Englee on the east coast, a Government nurse at Port Saunders, and two Mission nurses at Flower's Cove on the west coast. The hospital ship Strathcona with a doctor and a dentist visited every settlement in the White Bay district during the summer, and removed to hospital all persons needing in-patient treatment or special supervision. Dr. T. G. Hood describes the work of the Harrington Hospital, where pulmonary tuberculosis is the outstanding problem. It is remarked that thyroid disease is extremely rare in Labrador so far as the coast is concerned, though more common in the interior. The general interest of the report is enhanced by an account of the industrial handicrafts work of the Mission, which is of great value economically and provides occupation for the long winter periods. Reference is repeatedly made to the friendliness, cheeriness under difficulties, and gratitude of these people.

\section{Use and Abuse of Antiseptics}

Dr. A. C. F. HALFord writes from Brisbane: I have been a laboratory worker and a clinical surgeon and 1 try to hold my experience in both spheres in their proper perspectives. It is from both points of view that I have disagreed with Mr. Norman C. Lake (Journal, April 2, p. 753), and I am convinced that neither laboratory tests nor clinical use justify us in giving spirit solutions of biniodide of mercury a place anywhere. Especially is this so in minor surgery. My real purpose in writing on any phase of surgical and midwifery practice is to draw serious attention to the undue prevalence of sepsis in spite of Mr. Lake's claims of the hundreds of thousands of operations he mentions. The surgeon who uses only hot water and soap has been known to make the same claim, but there are many wounds that break down, and the fault is not merely the choice of antiseptic; many are chosen but few used wisely. It is the protection of a wound against exogenous infections-cross-infections and secondary infections-that worries us, a position to-day that is approached, by the younger practitioners especially, with hope but not with ertainty. Why does the laboratory worker not step in and that a wound dressed with no antiseptic protection at all cannot be safe any more than the soiled "sterile" plug of a tube of culture liquid can prevent contamination of the whole? Thus is it that I continue to write protesting against inefficient lotions and aseptic dressings and plead for a return to intelligent antiseptic measures.

\section{Ophthalmology in Palestine}

In spite of the unsettled conditions in Palestine the progress made by the Ophthalmic Hospital of St. John of Jerusalem in 1937 was most satisfactory. The number of new outpatients, 21,367, was the largest on record, and the outpatient attendances reached the remarkably high figure of 108,616. The number of in-patients, 907, was below that for the previous year. The annexe was as full as usual, and there was a daily average of 100 patients in the arcades of the Khan. Training in ophthalmology of the Palestinian nurses continued efficient and popular. It has been decided to build a new block for paying patients and for a physicotherapeutic department on the south side of the existing buildings, the appeal for funds having resulted in the receipt of over $£ 10,528$. Many patients were received from the Army and the Royal Air Force. A slight earthquake shock towards the end of the year under review opened up the cracks in the roofs, and all leaked badly during the November rains with the exception of the large dispensary roof, which had previously received special asphalt treatment. The question of treating all the roofs in this manner is now being considered carefully. Sir Horace Rumbold, Vice-Chairman of the Palestine Commission, described the hospital as "the finest piece of British propaganda in the East." The electric lighting, heating, and power supply of the institution has now been undertaken by the Jerusalem Electric Corporation at a reasonable flat rate. The civil disturbances in Palestine interfered with road transport and diminished the attendances by the peasantry, as contrasted with the local town population. The number and percentage of Jewish patients increased more particularly. The incidence of trachoma among the new patients, 85.82 per cent., was the lowest hitherto recorded at the hospital, indicating a temporary change in the character of the patients, there being more urban dwellers, among whom this disease is relatively less common. Some improvement was noted in the "blindness rate" in spite of a severe but localized outbreak of acute conjunctivitis affecting the townspeople. Potent factors in this outbreak were malnutrition, debilitation due to measles and whooping-cough, and the widespread economic distress consequent on unemployment. These cases do not respond well to treatment.

\section{Sedimentation Tests in General Practice}

Dr. A. E. FinckH (Sydney) writes: Any general practitioner wishing to employ the blood sedimentation test may find the following technique a great advantage: Use oxalated blood as for blood sugar examinations. Of a 10 per cent. potassium oxalate solution put one drop on the bottom of a small bottle; heat gently till evaporated to dryness. Keep a number of these in stock. Collect from 2 to $3 \mathrm{c} . \mathrm{cm}$. of blood from a vein and deposit in the "oxalate bottle"; shake gently. Take a graduated $1 \mathrm{c.cm}$. pipette and suck up to near the 0 mark and block with the finger. Block the other end by stabbing into a cake of soap or wax. Keep vertically at room temperature and read off in an hour.

\section{Medical Golf}

There were nearly a hundred competitors for the Manchester and District Medical Golfers' Association annual competition held on the Northenden links on May 25. The challenge cup for medal play was won by Dr. J. G. Stewart of Oldham with the best net score of 67. Dr. Stewart also won the Walter silver medal for the best gross score from a handicap of 10 or over. Dr. W. L. Hunter of Hale won the Walter gold medal with the best gross score of 73, and Dr. J. S. B. Mackay of Bury won the captain's second prize. A prize for the best net score over the first nine holes was won by Dr. J. E. R. Keyms of Fairfield, and another for the best score of the second nine holes by Dr. L. J. O'Loughlin of Moss Side. Play was oyer eighteen holes, and the weather was favourable.

\section{Corrigendum}

In Epitome paragraph No. 398 on sulphanilamide in cerebrospinal fever, which appeared in the Journal of May 14 at page 80, "The dosage was graduated from an upper limit of 15 grammes every four hours" should have read "The dosage was graduated from an upper limit of 15 grains every four hours." 